\title{
White Matter Microstructure Reflects Individual Differences in Music Reward Sensitivity
}

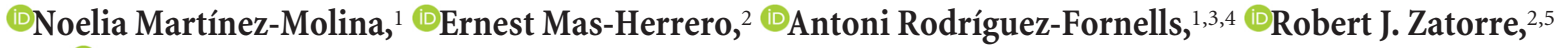 \\ and DJosep Marco-Pallarés ${ }^{1,3,6}$ \\ ${ }^{1}$ Department of Cognition, Development and Educational Psychology, University of Barcelona, L'Hospitalet de Llobregat, 08097 Barcelona, Spain, \\ ${ }^{2}$ Montreal Neurological Institute, McGill University, Montreal, Quebec H3A 2B4, Canada, ${ }^{3}$ Cognition and Brain Plasticity Group, Bellvitge Biomedical \\ Research Institute, L'Hospitalet de Llobregat, 08097 Barcelona, Spain, ${ }^{4}$ Institució Catalana de Recerca i Estudis Avançats, 08010 Barcelona, Spain,

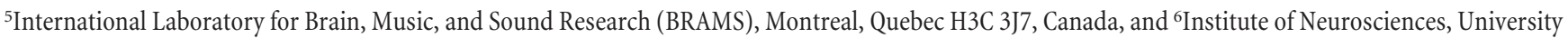 \\ of Barcelona, 08035 Barcelona, Spain
}

People show considerable variability in the degree of pleasure they experience from music. These individual differences in music reward sensitivity are driven by variability in functional connectivity between the nucleus accumbens (NAcc), a key structure of the reward system, and the right superior temporal gyrus (STG). However, it is unknown whether a neuroanatomical basis exists for this variability. We used diffusion tensor imaging and probabilistic tractography to study the relationship between music reward sensitivity and white matter microstructure connecting these two regions via the orbitofrontal cortex (OFC) in 38 healthy human participants (24 females and 14 males). We found that right axial diffusivity (AD) in the STG-OFC connectivity inversely correlated with music reward sensitivity. Additionally, right mean diffusivity and left $\mathrm{AD}$ in the NAcc-OFC tract also showed an inverse correlation. Further, AD in this tract also correlated with previously acquired BOLD activity during music listening, but not for a control monetary reward task in the NAcc. Finally, we used mediation analysis to show that AD in the NAcc-OFC tract explains the influence of NAcc activation during a music task on music reward sensitivity. Overall, our results provide further support for the idea that the exchange of information among perceptual, integrative, and reward systems is important for musical pleasure, and that individual differences in the structure of the relevant anatomical connectivity influences the degree to which people are able to derive such pleasure.

Key words: diffusion MRI; music; nucleus accumbens; probabilistic tractography; reward; white matter

Significance Statement

Music is one of the most important sources of pleasure for many people, but at the same time there are important individual differences in the sensitivity to musical reward. Previous studies have revealed the critical involvement of the functional connectivity between perceptual and subcortical brain areas in the enjoyment of music. However, it is unknown whether individual differences in music sensitivity might arise from variability in the structural connectivity among these areas. Here we show that structural connectivity between supratemporal and orbitofrontal cortices, and between orbitofrontal and nucleus accumbens, predict individual differences in sensibility to music reward. These results provide evidence for the critical involvement of the interaction between the subcortical reward system and higher-order cortical areas in music-induced pleasure.

\section{Introduction}

Music is deemed to be one of the most enjoyable of human experiences (Tramo, 2001; Dubé and Le Bel, 2003). Indeed, listening to pleasurable music activates areas of the reward system (Blood and Zatorre, 2001; Brown et al., 2004; Koelsch et al., 2006; Salimpoor et al., 2011, 2013; Koelsch, 2014), including regions engaged 
by primary reinforcers such as food or sex (Sescousse et al., 2013). However, sensitivity to musical pleasure shows an important degree of individual differences in the healthy population, ranging from people who experience intense musical pleasure to $\sim 3-5 \%$ of people who are insensitive to it (despite normal perceptual ability), a condition known as specific musical anhedonia (MasHerrero et al., 2013, 2014). This variability is driven by differences in functional connectivity between the right superior temporal gyrus (STG) and nucleus accumbens (NAcc) when listening to music (Martínez-Molina et al., 2016). Functional and structural connectivity are intrinsically related since the temporal correlation of activity across regions ultimately depends on the efficient information transfer mediated by structural pathways. However, little is known about the influence of the microstructural properties of these pathways on individual differences in music reward sensitivity. Therefore, to address this question we investigated the structural connectivity between the STG and the NAcc guided by our previous functional connectivity results (Martínez-Molina et al., 2016). However, there are limited direct anatomical connections between these two regions, since the STG connects largely to the caudate and putamen (Yeterian and Pandya, 1998), and therefore it is more likely that music information processed by the STG may access the NAcc indirectly via a third region. The orbitofrontal (OFC) is an ideal candidate to mediate the interaction between STG and NAcc (Haber, 2011) for several reasons, as follows: (1) it acts as a hub for multisensory integration including auditory information (Barbas, 1988, 1993; Romanski et al., 1999; Romanski and Goldman-Rakic, 2002; Rolls et al., 2006); (2) it is involved in reward valuation and subjective pleasantness (Kringelbach, 2005); (3) it shows increased functional connectivity with the NAcc as music gains reward value (Salimpoor et al., 2013); and (4) it sends projections to the central and lateral ventral striatum overlapping with the terminals that project to the NAcc (Haber and Knutson, 2010).

In the present study, we used probabilistic tractography (Behrens et al., 2007) to determine whether white matter microstructure between STG and OFC and between NAcc and OFC is associated with individual differences in music reward experience. Furthermore, to determine whether a structure-function relationship impacts such individual differences, we examined whether the contrast estimates of hemodynamic responses in the neuroanatomically defined NAcc obtained during a music task in the same participants from our previous study (Martínez-Molina et al., 2016) are related to the underlying white matter microstructure of both connectivities, as the anatomical hypothesis would predict. Finally, we performed mediation analysis to determine whether white matter microstructure might mediate the influence of the hemodynamic activity of the NAcc during a music task on behaviorally established music reward sensitivity, again as predicted by the anatomical model.

\section{Materials and Methods}

Participants. Diffusion tensor imaging (DTI) and T1 images were collected from a total of 43 right-handed nonmusician university students. Forty of these subjects were the same as those who participated in a previous fMRI study (Martínez-Molina et al., 2016). They completed the Barcelona Music Reward Questionnaire (BMRQ; Mas-Herrero et al., 2013), a validated psychometric tool to assess individual differences in music reward sensitivity. In addition, participants were evaluated for (1) global sensitivity to punishment and reward using the sensitivity to punishment and reward questionnaire (SPSRQ; Torrubia et al., 2001); (2) hedonia trait using the physical anhedonia scale (PAS; excluding those items referring to musical rewarding experiences to assess the hedonic impact of other activities or stimulus outside the music domain;
Chapman et al., 1976); and (3) amusia score using the Montreal Battery of Evaluation of Amusia (MBEA; Peretz et al., 2003). Based on the original cutoff values for the MBEA (Peretz et al., 2003), 5 participants with an overall MBEA score of $<78 \%$ were discarded, leading to a final sample size of 38 (mean age $=21.1$ years, $S D=3.8$ years; 24 females). All participants were healthy and free from any neurological or psychological disorders and gave written informed consent before participating in the study. All procedures were approved by the Ethics Committee of the Hospital Universitari de Bellvitge, Barcelona (PR181/13).

Data acquisition. Images were collected on a $3 \mathrm{~T}$ scanner (GE Discovery MR750w) using an eight-channel phased-array coil (GE Healthcare). High-resolution structural images [T1-weighted sequence (BRAVO); $\mathrm{TR}=11.668 \mathrm{~ms} ; \mathrm{TE}=4.79 \mathrm{~ms} ; \mathrm{TI}=450 \mathrm{~ms}$; flip angle $=12^{\circ}$; slice thickness $=1 \mathrm{~mm}$; matrix size $=512 \times 512$; field-of-view $(\mathrm{FOV})=280$ $\mathrm{mm}$ ] were recorded for anatomical reference. DTI data were acquired on the same scanner using a diffusion tensor spin-echo EPI sequence fully optimized for DT-MRI of white matter, with the following parameters: $\mathrm{TR}=12825 \mathrm{~ms}$; TE minimum, b-value $=1000 \mathrm{~s} / \mathrm{mm}^{2} ; 64$ diffusionweighted directions and 9 non-diffusion-weighted volumes; voxel size $=$ $2.0 \mathrm{~mm}^{3}$ (isotropic); matrix size $=128 \times 128 ; \mathrm{FOV}=256 \mathrm{~mm}$; 64 slices $2 \mathrm{~mm}$ thick and no gap.

DTI preprocessing. Diffusion data were processed using the Functional Magnetic Resonance Imaging of the Brain Software Library [FSL version 5.0, FMRIB (http://fsl.fmrib.ox.ac.uk/fsl)]. Eddy current-induced distortions and head motion displacements were corrected by affine registration of the 64 diffusion volumes to the first b0 volume using FSL (Jenkinson et al., 2002). The b-vector table (i.e., gradient directions) for each participant was rotated according to the parameters of this linear correction. The skull and other nonbrain tissue were removed using the FSL Brain Extraction Tool. Diffusivity measures [fractional anisotropy (FA); mean diffusivity (MD); axial diffusivity (AD)] were calculated by fitting a diffusion tensor model at each voxel to the diffusion data using Diffusion Toolkit software. Radial diffusivity (RD) was calculated as the mean of the second and third eigenvalues of the diffusion tensor.

Probabilistic tractography. We used probabilistic tractography to delineate the proposed tracts, as it is especially appropriate in the determination of the NAcc-OFC tract (accumbofrontal tract; Karlsgodt et al., 2015). Markov Chain Monte-Carlo sampling in the FSL BEDPOSTX tool (Behrens et al., 2003) was used to estimate within-voxel probability density functions of the principal diffusion direction. Based on these local probability density functions, probabilistic streamlines were computed using the FSL PROBTRACKX tool (Behrens et al., 2003), in which 5000 samples were taken for each input voxel with a 0.2 curvature threshold, $0.5 \mathrm{~mm}$ step length, 2000 steps/sample, and 0.01 for a subsidiary fiber volume fraction threshold.

Masks specification. Seed, waypoints, termination, and exclusion masks were defined for each path on the MNI152 T1 $1 \mathrm{~mm}$ template. We first defined the following three paths: one connecting the STG and NAcc; one connecting the STG and OFC; and another connecting the OFC and NAcc, within each hemisphere separately. The rationale for dissecting the path between the STG and NAcc was to confirm that no direct anatomical link existed between them as previous literature in monkeys had shown (Yeterian and Pandya, 1998). For this path, the exclusion mask included the entire contralateral hemisphere. For the structural connectivity between the STG and OFC, exclusion masks included the temporal pole, superior frontal regions, and regions posterior to the STG, including the occipital and parietal cortices. For the OFCNAcc tract, exclusion masks included the entire contralateral hemisphere and regions lateral to the putamen and posterior to the caudate. To quantify the overlap between the STG-OFC connectivity with anatomically dissected tracts passing through temporal and orbitofrontal regions, we also tracked the inferior fronto-occipital fasciculus (IFOF) and uncinated fasciculus (UNC). The exclusion masks included the contralateral hemisphere for both and also the internal capsule for the IFOF. The neuroanatomical masks were the Harvard-Oxford cortical and subcortical atlas-defined regions of interest (ROIs) of the NAcc (accumbens), STG (posterior division), OFC (frontal orbital cortex), TP (temporal pole), and OC (lateral occipital cortex, inferior division) included in the FSL package (Fig. 1A,B, anatomical masks, respectively, used for the 


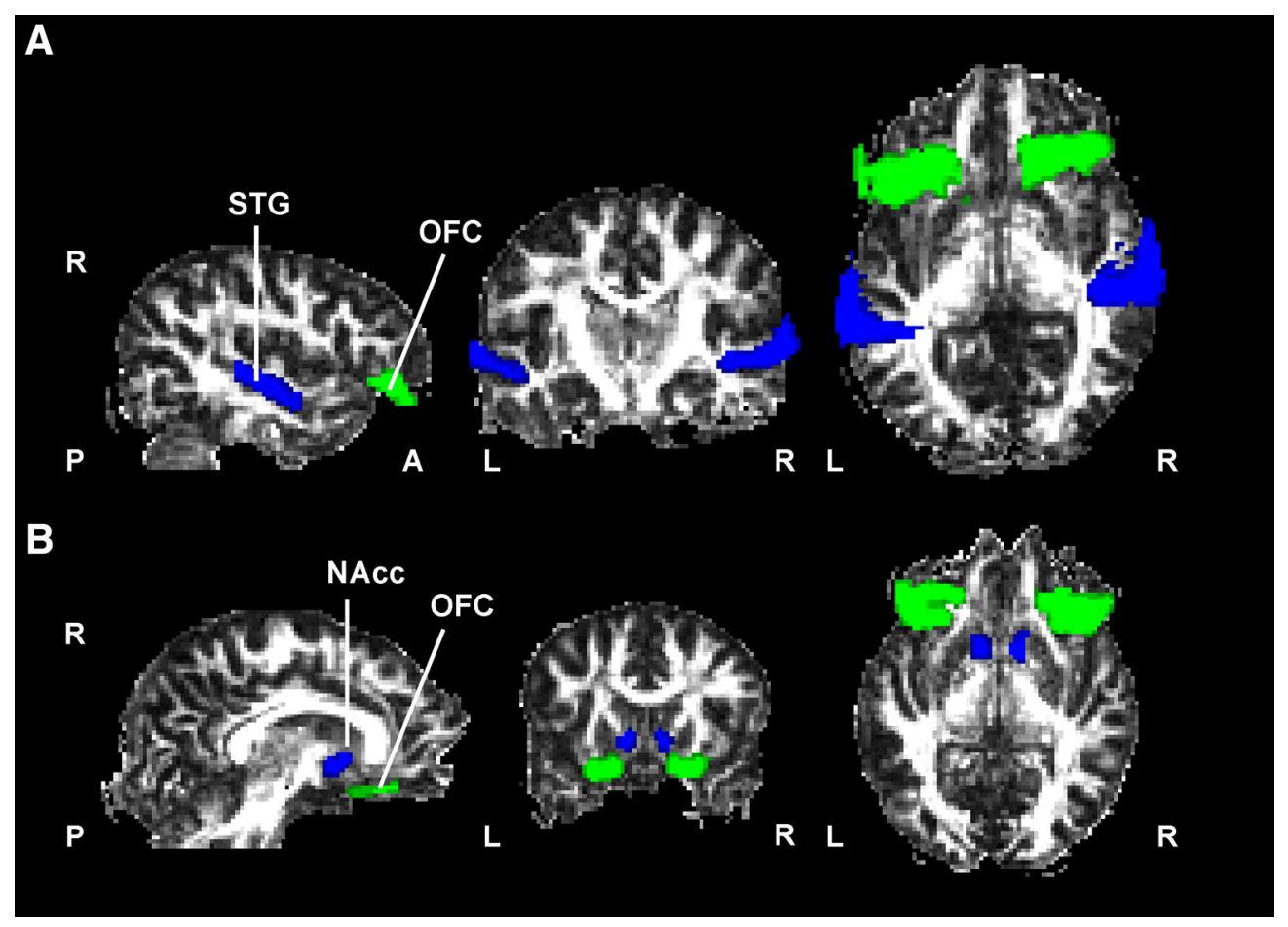

Figure 1. Neuroanatomical masks used for probabilistic tractography. $A, B$, Masks used in the fiber tracking of the STG-OFC $(\boldsymbol{A})$ and accumbofrontal tracts $(\boldsymbol{B})$ overlayed on a fractional anisotropy map from a representative subject in native space. Masks were extracted from the Harvard-0xford cortical and subcortical atlases (see Materials and Methods) and transformed to the native space of each subject. L, Left; $R$, right.

STG-OFC and NAcc-OFC connectivity distributions). The OFC was thresholded at $30 \%$ probability. Masks were normalized to each subject's diffusion space using the FMRIB Linear Image Registration Tool (Jenkinson and Smith, 2001) by applying the affine parameters obtained by coregistering the ROIs in MNI to the FMRIB58_FA template and next using nonlinear registration to convert ROIs to FA native space. Tractography was initiated from the STG as seed to the NAcc as a termination target, and then another tractography was performed in the opposite direction using the NAcc as seed and the STG as termination target. The same procedure applied to the tractography between the STG and OFC (Fig. 2A), between the OFC and NAcc (accumbofrontal tract; Fig. 2B), between the OC and OFC for the IFOF, and between the TP and OFC for the UC. The resulting paths were thresholded at a normalized probability value of 0.01 and visually inspected to confirm successful tracing in each individual subject based on the literature (Catani and Thiebaut de Schotten, 2008; Karlsgodt et al., 2015). The output images of individual connectivity distributions were binarized after normalizing by individual total counts of established samples (waytotal), dividing each voxel value by the waytotal number. The mean $\mathrm{FA}, \mathrm{MD}, \mathrm{AD}$, and $\mathrm{RD}$ were computed and averaged for the two directions in which tractography was performed for each path.

Experimental design and statistical analysis. A complete description of the music and gambling task used in the fMRI session can be found in the study by Martínez-Molina et al., 2016. Briefly, in the music task, participants listened to $1 \mathrm{~min}$ music excerpts while providing on-line pleasure ratings about the degree of pleasure they were experiencing. They indicated this by pressing a button in real time in a four-button magnetic resonance-compatible input device using a scale of $1-4(1=$ unpleasant, $2=$ low pleasure, $3=$ high pleasure, and $4=$ chill). Musical excerpts were intermixed with blocks of $30 \mathrm{~s}$ without sound. Two blocks of 8 musical excerpts each (4 pleasant and 4 unpleasant) were presented for a total of 16 musical pieces. In the gambling task, participants had to bet on one of two numbers (25 or 5 ) appearing on screen. After the selection, one number turned red and the other green. If the number selected by the participant turned green, the participant gained the corresponding amount of money in Euro cents; a red number indicated a monetary loss. In addition, in one-third of the trials, the selected number turned into a green or red 125 (boost gain or loss, respectively). Participants were given 10 euros and were encouraged to win as much money as possible. At the end of the scanning session, the final amount of money won by the participant was paid to him/her. Participants performed two runs of 90 trials each. Unknown to them, the result of each trial was pseudorandomly determined at the beginning of each run to ensure the same number of gain and loss trials and one-third or boost trials (half gains and half losses). Tasks were presented in counterbalanced order.

For each task, a GLM model was implemented in SPM8. In the music task, pleasant and unpleasant regressors were modeled time locked to $5 \mathrm{~s}$ after the onset of each musical excerpt. For the on-line music ratings, a separate first-order parametric regressor modeled the pleasure ratings from 1 to 4 . A rest regressor was also included with $30 \mathrm{~s}$ duration. In addition, separate regressors modeled the first $5 \mathrm{~s}$ of each musical excerpt and those pleasure ratings that were excluded (consecutive responses of the same pleasure rate and chills whose difference in latency was $<5 \mathrm{~s}$ ). In the gambling task, a regressor for trial onsets was defined time locked to the presentation of the monetary cue $(25,5$; or 5,25$)$. A first-order parametric regressor was included to model the reward magnitude and valence. Finally, a response regressor was included along with a firstorder parametric regressor modulating whether participants used the left or right button.

The contrast estimates for the parametric regressors of each task were computed by calculating the mean value from the contrast images (weighted $\beta$ values) within a neuroanatomically defined ROI of the NAcc (Hammers et al., 2003).

For each connectivity and hemisphere, we performed a linear stepwise regression analysis with the four DTI indices as independent variables and overall BMRQ scores as dependent variables in SPSS version 21. Subsequently, we performed additional linear stepwise regression analyses with the DTI scalars that were significant predictors of overall BMRQ scores. First, we conducted a stepwise regression analysis including BMRQ, SPSRQ, and PAS as independent variables. Second we used the contrast estimates of the hemodynamic response obtained from a music and a gambling task available from our previous fMRI study (MartínezMolina et al., 2016) as dependent variables in two separate stepwise linear regression analyses with the DTI scalars predicting music reward sensi- 


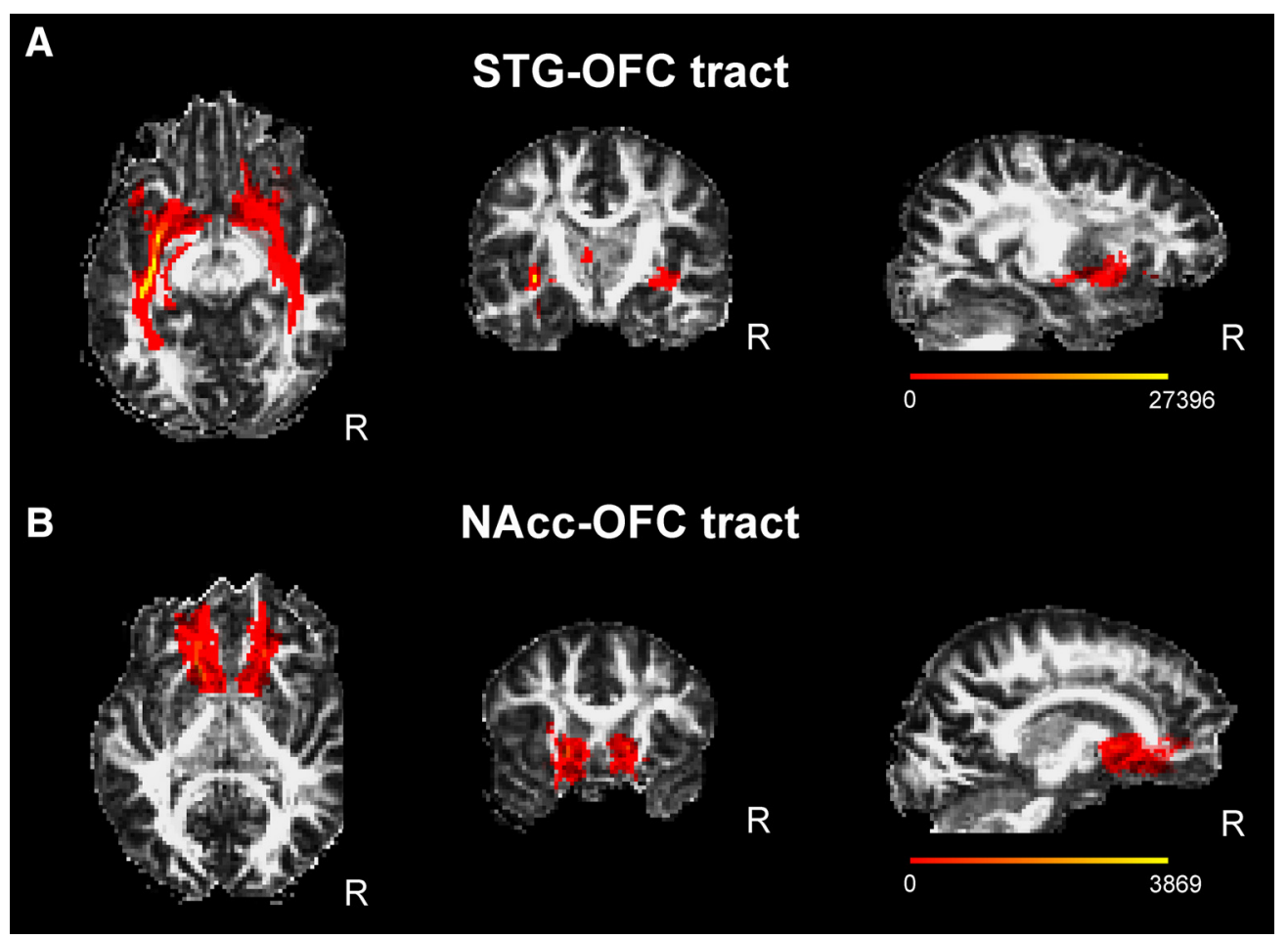

Figure 2. $\quad \boldsymbol{A}, \boldsymbol{B}, \mathrm{STG}-\mathrm{OFC}(\boldsymbol{A})$ and NACC-OFC $(\boldsymbol{B})$ tracts. Connectivity distributions are overlayed on a fractional anisotropy map in native space of a representative subject and averaged for both directions of tractography. Color bars display the range of connectivity values (number of samples from the seed that pass through a given voxel).

tivity as independent variables. In all stepwise regression analyses, the entry criterion was $p<0.05$ and the exit criterion was $p>0.10$. Tests for multicollinearity indicated that a very low level of multicollinearity was present in the analysis (variance inflation factors $<1.08$ and tolerances $>0.9$ )

To examine the structure-function relationship, we performed stepwise regression analyses with the DTI indices that significantly predicted music reward sensitivity and the BOLD signal contrast estimates from a NAcc ROI associated with the musical or monetary tasks from our previous publication (Martínez-Molina et al., 2016).

To control for multiple comparisons for the stepwise regression analyses performed, we used the FDR (false discovery rate) correction following the original procedure proposed by Benjamini and Hochberg (1995). The FDR is the expected proportion of rejected hypotheses that are mistakenly rejected in a family of hypotheses tests. We have reported the $p$ values followed by the FDR adjusted $p$ values ( $q$ values) at a desired FDR of 0.05 .

To assess the mediated effect, we defined single-level mediation analyses in MPlus version 7.11 (Muthén and Muthén, 1998) using maximum likelihood estimation. In addition, we used a bias-corrected bootstrap test, which provides confidence interval (CI) limits for indirect effects in mediation models. Bootstrapped confidence intervals are preferred over other available methods for testing mediation due to its lower type I error rate and high power to detect mediation (MacKinnon et al., 2004). Specifically, the indirect effect was tested using a bootstrap estimation approach with 1000 samples (Shrout and Bolger, 2002). To investigate the mediation of white matter architecture on the effect of NAcc BOLD responses on music reward sensitivity, we performed mediation analyses for $\mathrm{AD}$ and $\mathrm{MD}$ in each connectivity and hemisphere as well as mediation analyses for $\mathrm{AD}$ for the interaction of both connectivities computed as the product of $\mathrm{AD}$ values of the two connectivities in all possible combinations of right and left hemispheres.

\section{Results}

Participants presented a wide range of music reward sensitivity, as measured using the BMRQ scores ranging from high music hedonia (97) to low values associated with anhedonia (48). Based
Table 1. Psychometric information of participants $(N=38)$

\begin{tabular}{lllr}
\hline Pyschometric information & Mean (SD) & Other questionnaires & Mean (SD) \\
\hline BMRQ overall score & $75.3(15.1)$ & & \\
Musical seeking & $12.8(3.8)$ & Sensitivity to punishment & $8.5(4.6)$ \\
Emotion evocation & $15.4(3.9)$ & Sensitivity to reward & $9.7(4.1)$ \\
Mood regulation & $16.7(3.2)$ & PAS & $11.1(4.3)$ \\
Sensory-motor & $15.7(3.6)$ & MBEA & $89(5.6)$ \\
Social reward & $14.3(3.2)$ & Age & $21.1(3.8)$ \\
\hline
\end{tabular}

on their overall BMRQ scores and in the thresholds used in the study by Martínez-Molina et al. (2016), in the group there were 15 music hyperhedonic participants, 10 music hedonic participants, and 13 music anhedonic participants, allowing us to have a sufficient range of hedonic responses to pull out the relationship of interest with neuroanatomy. None of the participants showed low scores on general reward scales, thus ensuring that individual differences in reward processing were restricted to the music domain (Table 1).

To confirm that the STG and NAcc were not directly anatomically connected, as suggested by previous literature in nonhuman primates (Yeterian and Pandya, 1998), we first performed the probabilistic tractography between these two regions. Results from this tracking revealed that the number of streamlines between the STG and NAcc was almost negligible. Importantly, $73 \%$ and $63 \%$ of the participants had $<100$ streamlines in the STG-NAcc tract in the left and right hemispheres, respectively.

\section{STG-OFC connectivity}

The mean $\mathrm{AD}$ in the structural connectivity between the right STG and OFC correlated with music reward sensitivity $(r=$ $-0.43, r^{2}=0.18, F_{(1,37)}=8.07, p=0.007, q=0.035$; Fig. $\left.3 A\right)$. This relationship was negative, indicating that people with lower sensitivity to music reward show higher values for $\mathrm{AD}$ in the 
A

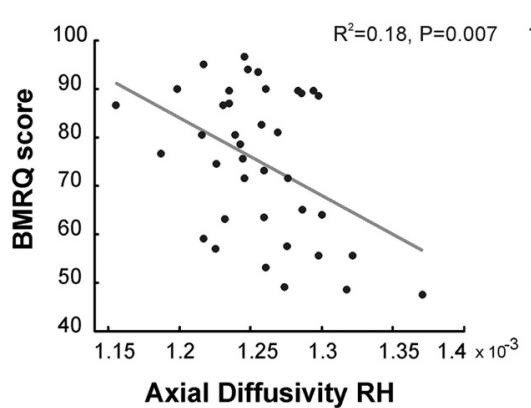

B

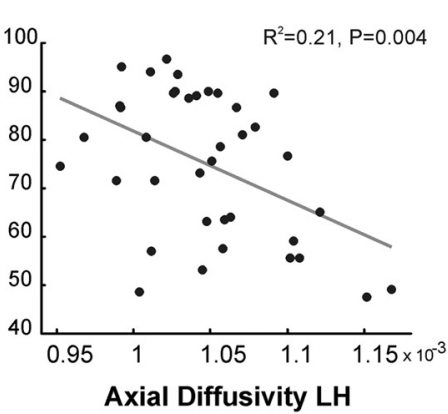

C NACC - OFC tract

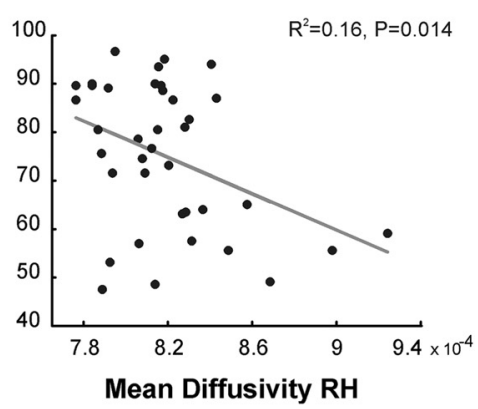

Figure 3. Microstructural properties of the STG-OFC and NAcc-OFC tracts with overall BMRQ scores. $A$, Right $A D$ values in the $S T G-O F C$ tract were inversely related to overall $B M R Q$ scores. $B, C$, Similar results in the $\mathrm{NAcc}-\mathrm{OFC}$ tract for left $\mathrm{AD}(\boldsymbol{B})$ and right $\mathrm{MD}(\boldsymbol{C})$ values. $\mathrm{AD}$ and $\mathrm{MD}$ are displayed at a different range of values for visualization purposes. $\mathrm{LH}$, Left hemisphere; $\mathrm{RH}$, right hemisphere.
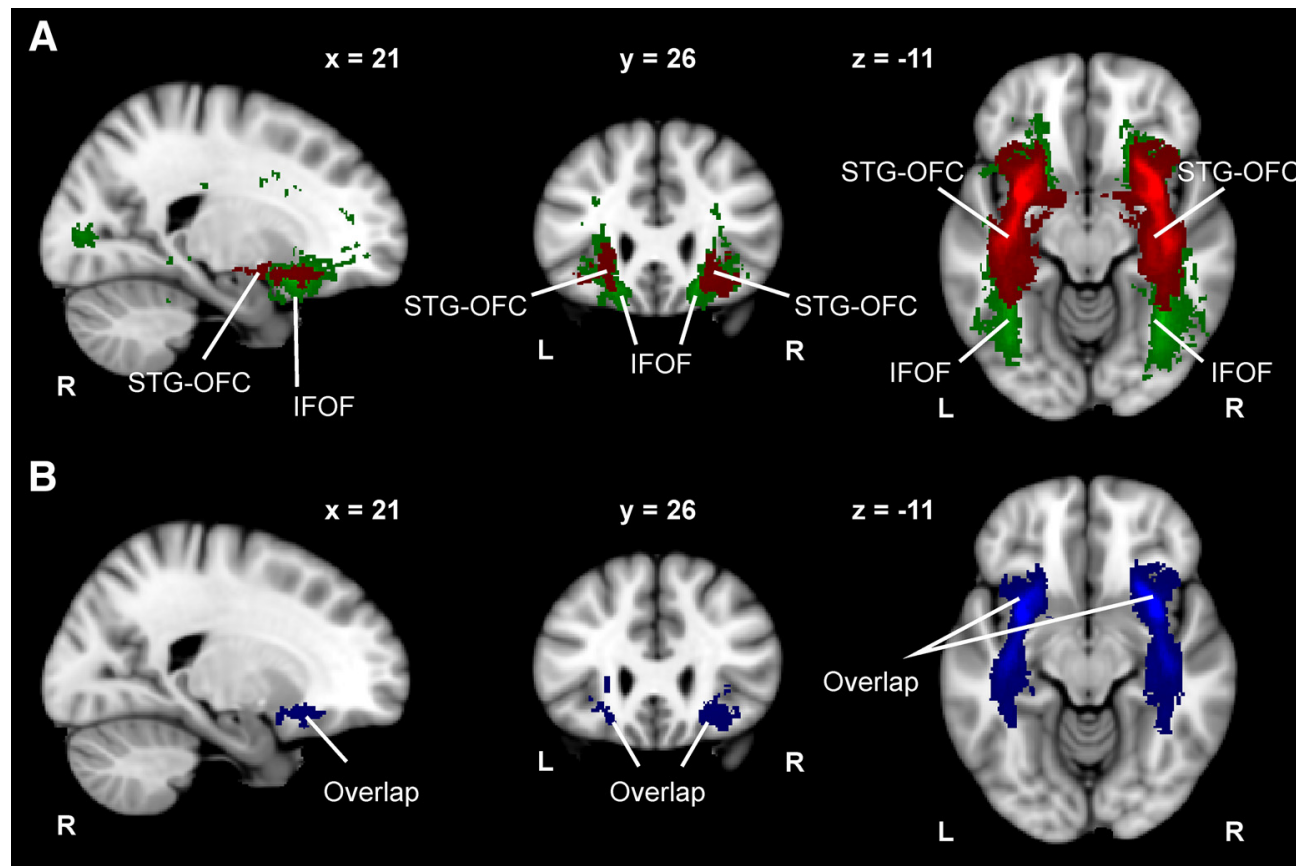

Figure 4. STG-OFC tract and IFOF comparison. $A$, Composite STG-OFC tract and IFOF computed by averaging the connectivity distributions of 38 subjects. $B$, Overlap between the STG-0FC tract and IFOF computed as the intersection between the connectivity distributions of 38 subjects. Neurological convention is used with MNI coordinates at the top of each slice. L, Left; R, right.

white matter connectivity between the right STG and OFC. We found no other DTI indices or volume (number of streamlines) in this connectivity predicting music reward sensitivity in the right hemisphere $(\mathrm{MD}, p=0.680 ; \mathrm{RD}, p=0.676 ; \mathrm{FA}, p=0.661$; volume, $p=0.107)$. No variables were entered into the model in the stepwise regression in the left hemisphere.

To rule out the contribution of general reward sensitivity to the observed effect, we performed another stepwise regression analysis where BMRQ, PAS, and SPSR overall scores were included as independent variables. Only the BMRQ emerged as a significant predictor of right $\mathrm{AD}$, indicating that this effect was specific for music reward sensitivity (BMRQ: $p=0.007, q=$ 0.028 ; PAS, $p=0.607$; Sensitivity to Punishment (SP), $p=0.649$; Sensitivity to Reward (SR), $p=0.471$ ).

The structural connectivity between the STG and OFC encompasses streamlines belonging to other anatomically identified tracts, mainly the IFOF and UNC, as both tracts have cortical terminations in the STG and OFC (Hau et al., 2016). We therefore determined the percentage of overlap with these tracts. We found that the STG-OFC tract shares most of its connectivity with the IFOF (Fig. $4 A, B$ ), whereas the spatial coincidence with the UNC was anecdotal. In the right hemisphere, the STG-OFC connectivity overlapped $66.24 \pm 1.95 \%$ (mean \pm SEM) with the IFOF and $0.17 \pm 0.05 \%$ with the UNC. In the left hemisphere, we found similar results: the STG-OFC connectivity overlapped $70.60 \pm 2.93 \%$ with the IFOF and $0.31 \pm 0.11 \%$ with the UNC. Importantly, given the fact that the IFOF showed a predominant overlap with the STG-OFC, we performed a stepwise regression analysis with DTI indices derived from this tract and found that none could predict overall BMRQ scores. Thus, music reward sensitivity is not subserved by the integrity of the entire tract, but more likely by the anterior and middle segments of the IFOF (Sarubbo et al., 2013), as illustrated in Figure 4.

\section{NAcc-OFC connectivity}

Having established the relationship between mean $\mathrm{AD}$ in the right STG-OFC connectivity and overall BMRQ scores, next we turned our attention to the structural connectivity between the 
OFC and NAcc. BMRQ scores correlated with both mean AD values in the left hemisphere $\left(r=-0.46, r^{2}=0.21, F_{(1,37)}=9.51\right.$, $p=0.004, q=0.020$; Fig. $3 B$ ), and mean MD values in the right hemisphere $\left(r=-0.40, r^{2}=0.16, F_{(1,37)}=6.69, p=0.014, q=\right.$ 0.070; Fig. 3C). No other significant relationship was observed between other DTI indices or tract volumes and overall BMRQ scores either in the left hemisphere $(\mathrm{MD}, p=0.775 ; \mathrm{RD}, p=$ 0.791; FA, $p=0.992$; vol., $p=0.430$ ) or right hemisphere (AD, $p=0.695$; RD, $p=0.593$; FA, $p=0.656$, vol.,$p=0.161$ ). Again, to confirm that this effect was specific to music reward sensitivity, we performed an additional stepwise regression analysis including BMRQ, PAS, and SPSR scores as independent variables. As expected, only the BMRQ emerged as a good predictor of right MD (BMRQ: $p=0.014, q=0.056$; PAS, $p=0.255 ;$ SP, $p=0.484$; SR, $p=0.765$ ) and left AD (BMRQ: $p=0.004, q=0.016$; PAS, $p=0.583 ; \mathrm{SP}, p=0.340 ; \mathrm{SR}, p=0.640)$, thus ruling out that this effect could be attributed to reward sensitivity more generally. These results converge with our findings from the STG-OFC connectivity.

\section{BOLD signal in the NAcc and NAcc-OFC} structural connectivity

To test whether structural connectivity is related to NAcc activation during music listening, we contrasted structural values showing significant relationship with music reward sensitivity with NAcc BOLD activity found in music and gambling tasks (Martínez-Molina et al., 2016). Linear stepwise regression analyses with the contrast estimates in the NAcc for the music and gambling tasks used as dependent variables in two separate analyses and $\mathrm{MD}$ and $\mathrm{AD}$ values as independent variables showed that the activity in the NAcc for musical pleasure was associated with mean $\mathrm{AD}$ in the left hemisphere $\left(r=-0.35, r^{2}=0.12, F_{(1,37)}=4.85\right.$, $p=0.034, q=0.068$; Fig. $5 A)$ and right hemisphere $\left(r=-0.46, r^{2}=\right.$ $0.21, F_{(1,37)}=9.67, p=0.004, q=0.008$; Fig. $\left.5 B\right)$ for the connectivity between the OFC and NAcc. Unlike AD, MD was not significantly associated with NAcc activation to musical pleasure (right hemisphere, $p=0.594$; left hemisphere, $p=0.073$ ). Further, neither MD nor $\mathrm{AD}$ values in this tract were entered into the model as predictors of activity in the NAcc in response to monetary reward. In the STGOFC connectivity, no significant relationship was observed in the stepwise regression between $\mathrm{MD}$ or $\mathrm{AD}$ and the contrast estimates of NAcc activity in the music or gambling task.

\section{Mediation analysis}

Mediation analysis is a method based on structural equation modeling that allows inferring causal relationships (Gunzler et al., 2013). In this study, we aimed to test whether lower NAcc activity during a music task leads to flattened hedonic responses to music (measured behaviorally with the BMRQ) via the interactions across regions that depend upon white matter connectivity (indirect or mediator variable), or whether the behavioral outcome can be explained more simply via a direct effect of activity on hedonic response. As expected, our findings indicated that hemodynamic activity during a music task in the NAcc was a significant predictor of music reward sensitivity $(b=7.007, p=$ 0.036). But, more importantly, we found a mediated, or indirect, effect for NAcc activity predicting music reward sensitivity through $\mathrm{AD}$ in the NAcc-OFC tract $(b=2.867,95 \% \mathrm{CI}=0.50-$ 7.33; Fig. 6A). In addition, NAcc activity was no longer a significant predictor of music reward sensitivity after controlling for $\mathrm{AD}(b=4.140, p=0.280)$, suggesting full mediation by microstructural architecture. Consistent with the results from the cor-
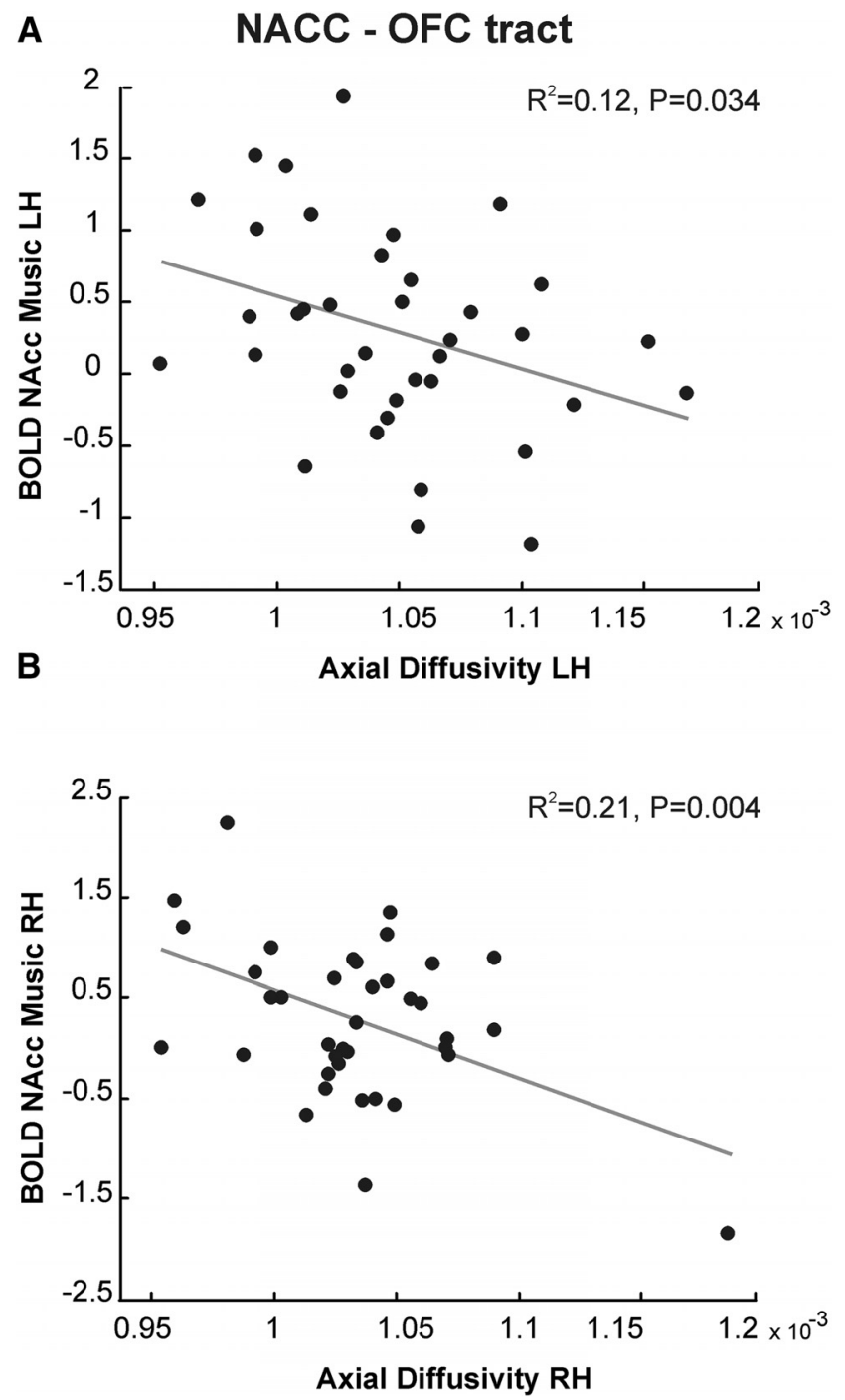

Figure 5. Structure-function relationship. $A$, Left $A D$ values in the $\mathrm{NAcc}-0 \mathrm{FC}$ tract predict hemodynamic activity in the left NAcc in response to musical pleasure. $\boldsymbol{B}$, Similar results for AD in the right hemisphere. LH, Left hemisphere, $\mathrm{RH}$, right hemisphere.

relation between overall $\mathrm{BMRQ}$ scores and $\mathrm{AD}$ in the NAcc-OFC connectivity, this effect was found in the left hemisphere. No mediation was found for $\mathrm{AD}$ in the NAcc-OFC connectivity in the right hemisphere or for MD in either hemisphere. This analysis yielded no significant results when repeated with right $\mathrm{AD}$ as a mediator in the STG-OFC connectivity.

In addition, we also included as mediator the interaction of $\mathrm{AD}$ in the STG-OFC and NAcc-OFC connectivity. We computed the mediation effect of the interaction between $\mathrm{AD}$ in the STG-OFC connectivity in the right hemisphere (where we found a significant relationship with overall BMRQ scores) with $\mathrm{AD}$ in the NAcc-OFC connectivity of the two hemispheres. The rationale for inclusion of the mediation of right STG-OFC connectivity with left NAcc-OFC connectivity was to consider that the input from the STG to the OFC in the right hemisphere can reach the contralateral OFC via interhemispheric connections. Remarkably, this was what we obtained in the mediation analysis. The indirect effect was significant only for the interaction between the right $\mathrm{AD}$ in the STG-OFC connectivity with the $\mathrm{AD}$ in the NAcc-OFC connectivity in the left hemisphere $(b=2.702$, $95 \% \mathrm{CI}=0.20-7.46$; Fig. $6 B)$ and right hemisphere $(b=3.047$, $95 \% \mathrm{CI}=0.03-7.36$; Fig. $6 \mathrm{C})$. This result is in agreement with 


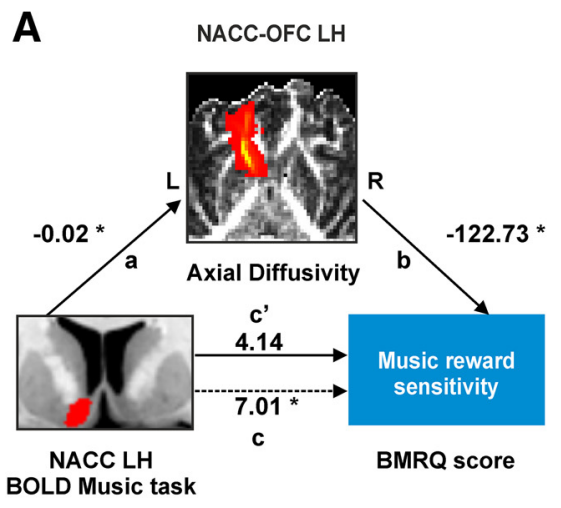

B STG-OFC RH x NACC-OFC LH

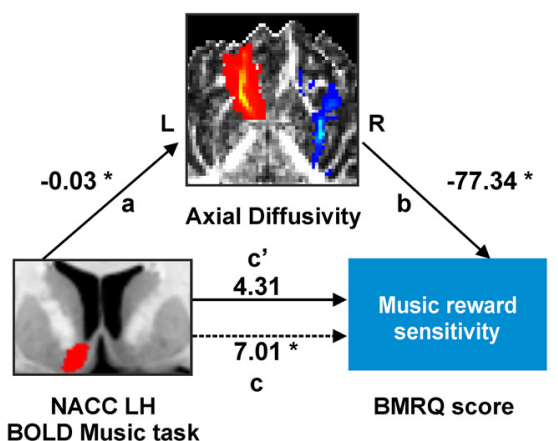

C STG-OFC RH x NACC-OFC RH

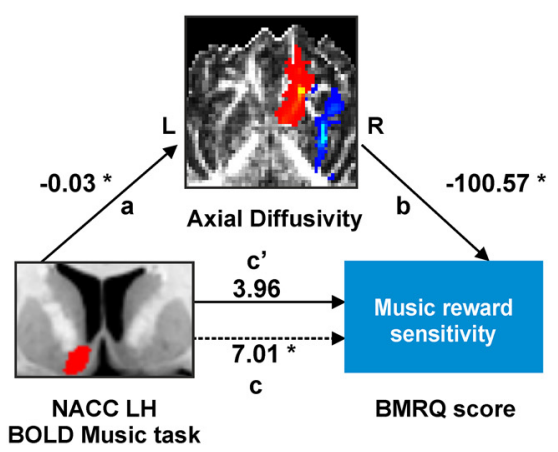

Figure 6. Structural connectivity mediates the effect of NAcc activation in response to musical pleasure on individual differences in music reward sensitivity. $A$, Mediation analysis demonstrating that the influence of NAcc activity during a music task on music reward sensitivity is mediated through left $A D$ in the NAcc-OFC tract. Path a shows coefficients for the effect of left NAcc activity on left $A D$ in the NAcc-OFC tract. Path b shows the coefficients for the effect of left $A D$ in the NAcc-OFC tract on music reward sensitivity. Paths $c$ and $c^{\prime}$ show coefficients for the total (dashed line) and direct (solid line) effects of left NAcc activity on music reward sensitivity. Significant relationships are denoted by asterisks $\left(^{*}\right): p<0.05 .95 \% \mathrm{Cl}(0.50-7.33)$ for the indirect effect. $B, C$, Similar results for the interaction of right $A D$ in the STG-OFC tract with either left $(\boldsymbol{B})$ or right $(\boldsymbol{C}) \mathrm{AD}$ in the $\mathrm{NACC}-\mathrm{OFC}$ tract. $95 \% \mathrm{Cl}(0.20-7.46)$ for the indirect effect of the interaction between right $A D$ in the STG-OFC tract and left $A D$ in the NAcc-OFC tract. $95 \%$ $\mathrm{CI}(0.03-7.36)$ for the indirect effect of the interaction between right AD in the STG-OFC tract and right $A D$ in the NACC $-O F C$ tract. The bottom-left inset $(A-C)$ represents the left NAcc ROIs from a neuroanatomical atlas (Hammers et al., 2003). The middle inset represents the left $\mathrm{NAcc}-\mathrm{OFC}$ tract $(\boldsymbol{A})$, the right STG-OFC and left NAcc-OFCtracts $(\boldsymbol{B})$, and the right STG-OFC and right NAcc-OFC tracts $(C)$ overlayed on a fractional anisotropy map in native space of a representative subject (same subject from $\boldsymbol{A}$ to $\boldsymbol{C}$ ) and averaged for both directions of tractography. LH, Left hemisphere, RH, right hemisphere.

our notion that the bilateral OFC may serve as a waystation for auditory information to gain access to the reward system and, further, that white matter connectivity may mediate the effect of NAcc activity on music reward sensitivity.

\section{Discussion}

In the present study, we demonstrated that white matter microstructure of connectivity distributions between STG-OFC and NAcc-OFC is related to individual differences in music reward processing. This relationship was not explained by general reward sensitivity or by sensitivity to punishment or reward, as measured through standard scales. In addition, we found that NAcc BOLD activity during music listening predicted such individual differences through the mediation of the structural connectivity among the STG, the OFC, and the NAcc. These results show the critical involvement of the white matter microstructure of frontotemporal and frontostriatal tracts in music reward processing.

The first structural connectivity involved in individual differences associated with music reward sensitivity was the one connecting the STG and the OFC. Although the projections from auditory cortex to OFC have been well documented in tracing studies in primates (Barbas, 1988; Morecraft et al., 1992; Romanski et al., 1999; Haber et al., 2006), the STG-OFC tract as such has not been histologically dissected in the human brain and is probably composed by the intermingling of fiber bundles passing through these regions. Indeed, we found a strong overlap $(>60 \%)$ of this connectivity with the IFOF, whereas the spatial coincidence between the STG-OFC and the UNC was anecdotal $(<1 \%)$. Moreover, stepwise regression analyses did not yield any DTI index from the IFOF as a predictor of overall BMRQ scores. Therefore, we speculate that individual differences in music reward sensitivity may be associated with white matter microstructure of the anterior and middle segments of the IFOF (Sarubbo et al., 2013). Future studies may consider quantification approaches that divide the IFOF into different profiles (Yeatman et al., 2012) or subcomponents (Wu et al., 2016) along its trajectory to demonstrate that the anterior and middle portions may be more sensitive to music reward processes. Interestingly, in the STG-OFC connectivity we found a relationship between white matter microstructure and BMRQ scores only in the right hemisphere. This would support the hemispheric lateralization hypothesis ( $\mathrm{Za}$ torre et al., 2002) assigning a predominant role of right auditory cortices in many aspects of tonal and musical processing (Patterson et al., 2002; Hyde et al., 2008; Albouy et al., 2013; Cha et al., 2016; Coffey et al., 2016). The importance of the right STG in this result is in keeping with a similar functional connectivity asymmetry in the same population from the previous study (MartínezMolina et al., 2016).

The second structural tract analyzed in the present study was the NAcc-OFC. This tract was defined following the procedure for the probabilistic tractography dissection of the accumbofrontal tract described by Karlsgodt et al. (2015). The accumbofrontal tract mainly connects the NAcc core with projections from the medial aspects of the OFC and has been identified postmortem in the human brain (Rigoard et al., 2011). This tract has also been independently dissected using tracing techniques in nonhuman primates (Haber et al., 1995) and deterministic tractography in humans (Samanez-Larkin et al., 2012). Neuroanatomically, the accumbofrontal is more medial than the adjacent anterior thalamic radiation or UNC, as well as being superior to the latter (Peters et al., 2014; Karlsgodt et al., 2015). Interestingly, restingstate analyses suggest a differential connectivity pattern of the inferior ventral striatum (i.e., the NAcc) with medial regions of the OFC that could be mediated by this structural connection (Di Martino et al., 2008). Our structural results are in line with those 
of previous studies showing that the functional connectivity between the NAcc and OFC is important in music listening (Menon and Levitin 2005) and to assign reward value to music (Salimpoor et al., 2013). Furthermore, our findings are also consistent with those of Alluri et al. (2015), who showed that left NAcc functional connectivity could be more sensitive to individual differences in music reward processing during music listening. However, it is important to note that the direction of the connectivity is not reflected in standard functional connectivity or tractography analyses. Further studies using effective connectivity measures such as dynamic causal modeling (DCM; Friston et al., 2003) are needed to understand better the directionality of such interactions (for an example of the use of DCM to study the interaction among auditory cortex, basal ganglia, and ventral striatum in rhythm perception, see Brodal et al., 2017).

Collectively, the results from both white matter connectivities analyzed in the present study provide converging evidence for our previous functional connectivity findings showing that specific musical anhedonia (the lack of musical pleasure in the absence of general anhedonia, amusia, or impairment of emotion recognition) is associated with decreased functional connectivity between the right STG and NAcc (Martínez-Molina et al., 2016). Interestingly, a previous study (Sachs et al., 2016) showed that people who experienced chills with music presented an increased structural connectivity between the STG and the insula and mPFC bilaterally compared with those that do not. In addition, in a single case study of a person with individual anhedonia, Loui et al. (2017) also found reduced tract volume and increased FA between the auditory and reward-related regions. In this context, the present anatomical results contribute to prior functional connectivity studies suggesting that information transfer among the perceptual, integrative, and reward systems may facilitate hedonic experiences with music. It is important to note that approximately one-third of our participants were individuals with specific musical anhedonia. The inclusion of this group increases the range of hedonic responses, allowing us to pull out the relationships of interest in the anatomy.

Regarding the patterns found for diffusivity parameters in relation to music reward sensitivity, $\mathrm{AD}$ consistently emerged as a significant predictor of BMRQ overall scores in all the analyses. $\mathrm{AD}$ corresponds to the largest eigenvalue in the diffusion tensor ellipsoid and describes the diffusion in the principal direction of water movement parallel to the fiber tract. Initial animal studies showed an AD decrease in response to axonal damage and loss of axonal integrity following acute neuronal damage (Kim et al., 2006; Sun et al., 2008; Budde et al., 2009). However, further studies have shown that during the chronic injury stage this pattern may reverse, with $\mathrm{AD}$ values increasing rather than decreasing (for review, see Aung et al., 2013). Interestingly, the relationship we found between DTI indices and overall BMRQ scores was negative. In other words, individuals with low music reward sensitivity were characterized by higher AD in both the STG-OFC and the NAcc-OFC connectivities. Previous studies in other cognitive domains have shown a similar inverse relationship between $\mathrm{AD}$ and behavior (e.g., in executive function and reinforcement learning tasks; Koch et al., 2010; Fjell et al., 2011; Borghesani et al., 2013; Alm et al., 2015). Importantly, Wang et al. (2017) reported increased mean and axial diffusivity in the corpus callosum, right longitudinal fasciculus, and right IFOF in people with congenital amusia, a condition characterized by difficulties in music perception of different acoustic features including pitch. These results together with our current findings, indicate that better musical perception and enjoyment would be associated with reduced axial and mean diffusivity in different white matter connections, with special importance of the IFOF. However, note that while amusia was related to $\mathrm{AD}$ increases in the posterior segment of the IFOF, in the current study musical reward was related to anterior and medial segments connecting with the OFC. Wang et al. (2017) proposed that increases of MD and AD might reflect reduced cell density which in turn would support an impairment of the affected tracts in people with amusia. However, although previous literature indicates that $\mathrm{AD}$ may be a sensitive index of white matter integrity, it still remains unclear what cellular mechanisms are reflected by long-term changes in AD (Zatorre et al., 2012).

In conclusion, our results demonstrate that hedonic individual differences in music reward sensitivity are associated with white matter microstructure in the STG-OFC and NAcc-OFC connectivities. Results of this study provide neuroanatomical support for the idea that exchange of information among perceptual, integrative, and reward systems through functional and structural connectivity is crucial in the process of deriving pleasure from music. Our findings also have important implications for understanding the structural correlates of specific musical anhedonia and should inform prospective tractography studies in specific anhedonias (and hyperhedonias) in other domains, opening the possibility of using MD and AD values in the STGOFC and NAcc-OFC structural connectivity as neuroanatomical markers of individual differences in music reward sensitivity to guide clinical studies using music-based interventions.

\section{References}

Albouy P, Mattout J, Bouet R, Maby E, Sanchez G, Aguera PE, Daligault S, Delpuech C, Bertrand O, Caclin A, Tillmann B (2013) Impaired pitch perception and memory in congenital amusia: the deficit starts in the auditory cortex. Brain 136:1639-1661.

Alluri V, Brattico E, Toiviainen P, Burunat I, Bogert B, Numminen J, Kliuchko M (2015) Musical expertise modulates functional connectivity of limbic regions during continuous music listening. Psychomusicol Music Mind Brain 25:443-454.

Alm KH, Rolheiser T, Mohamed FB, Olson IR (2015) Fronto-temporal white matter connectivity predicts reversal learning errors. Front Hum Neurosci 9:343.

Aung WY, Mar S, Benzinger TL (2013) Diffusion tensor MRI as a biomarker in axonal and myelin damage. Imaging Med 5:427-440.

Barbas H (1988) Anatomic organization of basoventral and mediodorsal visual recipient prefrontal regions in the rhesus monkey. J Comp Neurol 276:313-342.

Barbas H (1993) Organization of cortical afferent input to orbitofrontal areas in the rhesus monkey. Neuroscience 56:841-864.

Behrens TEJ, Berg HJ, Jbabdi S, Rushworth MFS, Woolrich MW (2007) Probabilistic diffusion tractography with multiple fibre orientations: What can we gain? NeuroImage 34:144-155.

Behrens TE, Woolrich MW, Jenkinson M, Johansen-Berg H, Nunes RG, Clare S, Matthews PM, Brady JM, Smith SM (2003) Characterization and propagation of uncertainty in diffusion-weighted MR imaging. Magn Reson Med 50:1077-1088.

Benjamini Y, Hochberg Y (1995) Controlling the false discovery rate: a practical and powerful approach to multiple testing. J R Stat Soc Series B Stat Methodol 57:289-300.

Blood AJ, Zatorre RJ (2001) Intensely pleasurable responses to music correlate with activity in brain regions implicated in reward and emotion. Proc Natl Acad Sci U S A 98:11818-11823.

Borghesani PR, Madhyastha TM, Aylward EH, Reiter MA, Swarny BR, Schaie KW, Willis SL (2013) The association between higher order abilities, processing speed, and age are variably mediated by white matter integrity during typical aging. Neuropsychologia 51:1435-1444.

Brodal HP, Osnes B, Specht K (2017) Listening to rhythmic music reduces connectivity within the basal ganglia and the reward system. Front Neurosci 11:153.

Brown S, Martinez MJ, Parsons LM (2004) Passive music listening sponta- 
neously engages limbic and paralimbic systems. Neuroreport 15:20332037.

Budde MD, Xie M, Cross AH, Song SK (2009) Axial diffusivity is the primary correlate of axonal injury in the experimental autoimmune encephalomyelitis spinal cord: a quantitative pixelwise analysis. J Neurosci 29:2805-2813.

Catani M, Thiebaut de Schotten M (2008) A diffusion tensor imaging tractography atlas for virtual in vivo dissections. Cortex 44:1105-1132.

Cha K, Zatorre RJ, Schönwiesner M (2016) Frequency selectivity of voxelby-voxel functional connectivity in human auditory cortex. Cereb Cortex 26:211-224.

Chapman LJ, Chapman JP, Raulin ML (1976) Scales for physical and social anhedonia. J Abnorm Psychol 85:374-382.

Coffey EB, Herholz SC, Chepesiuk AM, Baillet S, Zatorre RJ (2016) Cortical contributions to the auditory frequency-following response revealed by MEG. Nat Commun 7:11070.

Di Martino A, Scheres A, Margulies DS, Kelly AM, Uddin LQ, Shehzad Z, Biswal B, Walters JR, Castellanos FX, Milham MP (2008) Functional connectivity of human striatum: a resting state fMRI study. Cereb Cortex 18:2735-2747.

Dubé L, Le Bel J (2003) The categorical structure of pleasure. Cogn Emot 17:263-295.

Fjell AM, Westlye LT, Amlien IK, Walhovd KB (2011) Reduced white matter integrity is related to cognitive instability. J Neurosci 31:18060-18072.

Friston KJ, Harrison L, Penny W (2003) Dynamic causal modelling. Neuroimage 19:1273-1302.

Gunzler D, Chen T, Wu P, Zhang H (2013) Introduction to mediation analysis with structural equation modeling. Shanghai Arch Psychiatry 25: 390-394.

Haber SN (2011) Neuroanatomy of reward: a view from the ventral striatum. In: Neurobiology of sensation and reward, Chap 11 (Gottfried JA, ed), pp 235-262. Boca Raton, FL: CRC/Taylor and Francis.

Haber SN, Knutson B (2010) The reward circuit: linking primate anatomy and human imaging. Neuropsychopharmacology 35:4-26.

Haber SN, Kunishio K, Mizobuchi M, Lynd-Balta E (1995) The orbital and medial prefrontal circuit through the primate basal ganglia. J Neurosci 15:4851-4867.

Haber SN, Kim KS, Mailly P, Calzavara R (2006) Reward-related cortical inputs define a large striatal region in primates that interface with associative cortical connections, providing a substrate for incentive-based learning. J Neurosci 26:8368-8376.

Hammers A, Allom R, Koepp MJ, Free SL, Myers R, Lemieux L, Mitchell TN, Brooks DJ, Duncan JS (2003) Three-dimensional maximum probability atlas of the human brain, with particular reference to the temporal lobe. Hum Brain Mapp 19:224-247.

Hau J, Sarubbo S, Perchey G, Crivello F, Zago L, Mellet E, Jobard G, Joliot M, Mazoyer BM, Tzourio-Mazoyer N, Petit L (2016) Cortical terminations of the inferior fronto-occipital and uncinate fasciculi: anatomical stembased virtual dissection. Front Neuroanat 10:58.

Hyde KL, Peretz I, Zatorre RJ (2008) Evidence for the role of the right auditory cortex in fine pitch resolution. Neuropsychologia 46:632-639.

Jenkinson M, Smith S (2001) A global optimisation method for robust affine registration of brain images. Med Image Anal 5:143-156.

Jenkinson M, Bannister P, Brady M, Smith S (2002) Improved optimization for the robust and accurate linear registration and motion correction of brain images. Neuroimage 17:825-841.

Karlsgodt KH, John M, Ikuta T, Rigoard P, Peters BD, Derosse P, Malhotra AK, Szeszko PR (2015) The accumbofrontal tract: diffusion tensor imaging characterization and developmental change from childhood to adulthood. Hum Brain Mapp 36:4954-4963.

Kim JH, Budde MD, Liang HF, Klein RS, Russell JH, Cross AH, Song SK (2006) Detecting axon damage in spinal cord from a mouse model of multiple sclerosis. Neurobiol Dis 21:626-632.

Koch K, Wagner G, Dahnke R, Schachtzabel C, Güllmar D, Reichenbach JR, Schlösser RG (2010) Structure-function relationships in the context of reinforcement-related learning: a combined diffusion tensor imagingfunctional magnetic resonance imaging study. Neuroscience 168:190199.

Koelsch S (2014) Brain correlates of music-evoked emotions. Nat Rev Neurosci 15:170-180.
Koelsch S, Fritz T, V Cramon DY, Müller K, Friederici AD (2006) Investigating emotion with music: an fMRI study. Hum Brain Mapp 27: 239-250.

Kringelbach ML (2005) The human orbitofrontal cortex: linking reward to hedonic experience. Nat Rev Neurosci 6:691-702.

Loui P, Patterson S, Sachs ME, Leung Y, Zeng T, Przysinda E (2017) White matter correlates of musical anhedonia: implications for evolution of music. Front Psychol 8:1664.

MacKinnon DP, Lockwood CM, Williams J (2004) Confidence limits for the indirect effect: distribution of the product and resampling methods. Multivar Behav Res 39:99-128.

Martínez-Molina N, Mas-Herrero E, Rodríguez-Fornells A, Zatorre RJ, Marco-Pallarés J (2016) Neural correlates of specific musical anhedonia. Proc Natl Acad Sci U S A 113:E7337-E7345.

Mas-Herrero E, Marco-Pallares J, Lorenzo-Seva U, Zatorre RJ, RodriguezFornells A (2013) Individual differences in music reward experiences. Music Percept 31:118-138.

Mas-Herrero E, Zatorre RJ, Rodriguez-Fornells A, Marco-Pallarés J (2014) Dissociation between musical and monetary reward responses in specific musical anhedonia. Curr Biol 24:699-704.

Menon V, Levitin DJ (2005) The rewards of music listening: response and physiological connectivity of the mesolimbic system. Neuroimage 28: 175-184.

Morecraft RJ, Geula C, Mesulam MM (1992) Cytoarchitecture and neural afferents of orbitofrontal cortex in the brain of the monkey. J Comp Neurol 323:341-358.

Muthén LK, Muthén B (1998) MPlus user’s guide. Los Angeles: Muthén and Muthén.

Patterson RD, Uppenkamp S, Johnsrude IS, Griffiths TD (2002) The processing of temporal pitch and melody information in auditory cortex. Neuron 36:767-776.

Peretz I, Champod AS, Hyde K (2003) Varieties of musical disorders. The Montreal battery of evaluation of amusia. Ann N Y Acad Sci 999:58-75.

Peters BD, Ikuta T, DeRosse P, John M, Burdick KE, Gruner P, Prendergast DM, Szeszko PR, Malhotra AK (2014) Age-related differences in white matter tract microstructure are associated with cognitive performance from childhood to adulthood. Biol Psychiatry 75:248-256.

Rigoard P, Buffenoir K, Jaafari N, Giot JP, Houeto JL, Mertens P, Velut S, Bataille B (2011) The accumbofrontal fasciculus in the human brain. Neurosurgery 68:1102-1111.

Rolls ET, Critchley HD, Browning AS, Inoue K (2006) Face-selective and auditory neurons in the primate orbitofrontal cortex. Exp Brain Res 170: $74-87$.

Romanski LM, Goldman-Rakic PS (2002) An auditory domain in primate prefrontal cortex. Nat Neurosci 5:15-16.

Romanski LM, Tian B, Fritz J, Mishkin M, Goldman-Rakic PS, Rauschecker JP (1999) Dual streams of auditory afferents target multiple domains in the primate prefrontal cortex. Nat Neurosci 2:1131-1136.

Sachs ME, Ellis RJ, Schlaug G, Loui P (2016) Brain connectivity reflects human aesthetic responses to music. Soc Cogn Affect Neurosci 11:884891.

Salimpoor VN, Benovoy M, Larcher K, Dagher A, Zatorre RJ (2011) Anatomically distinct dopamine release during anticipation and experience of peak emotion to music. Nat Neurosci 14:257-262.

Salimpoor VN, van den Bosch I, Kovacevic N, McIntosh AR, Dagher A, Zatorre RJ (2013) Interactions between the nucleus accumbens and auditory cortices predict music reward value. Science 340:216-219.

Samanez-Larkin GR, Levens SM, Perry LM, Dougherty RF, Knutson B (2012) Frontostriatal white matter integrity mediates adult age differences in probabilistic reward learning. J Neurosci 32:5333-5337.

Sarubbo S, De Benedictis A, Maldonado IL, Basso G, Duffau H (2013) Frontal terminations for the inferior fronto-occipital fascicle: anatomical dissection, DTI study and functional considerations on a multi-component bundle. Brain Struct Funct 218:21-37.

Sescousse G, Caldú X, Segura B, Dreher JC (2013) Processing of primary and secondary rewards: a quantitative meta-analysis and review of human functional neuroimaging studies. Neurosci Biobehav Rev 37:681-696.

Shrout PE, Bolger N (2002) Mediation in experimental and nonexperimental studies: new procedures and recommendations. Psychol Methods 7:422-445.

Sun SW, Liang HF, Cross AH, Song SK (2008) Evolving wallerian degener- 
ation after transient retinal ischemia in mice characterized by diffusion tensor imaging. Neuroimage 40:1-10.

Torrubia R, Ávila C, Moltó J, Caseras X (2001) The sensitivity to punishment and sensitivity to reward questionnaire (SPSRQ) as a measure of Gray's anxiety and impulsivity dimensions. Personal Individ Differ 31: $837-862$.

Tramo MJ (2001) Biology and music. music of the hemispheres. Science 291:54-56.

Wang J, Zhang C, Wan S, Peng G (2017) Is congenital amusia a disconnection syndrome? A study combining tract- and network-based analysis. Front Hum Neurosci 11:473.

Wu Y, Sun D, Wang Y, Wang Y (2016) Subcomponents and connectivity of the inferior fronto-occipital fasciculus revealed by diffusion spectrum imaging fiber tracking. Front Neuroanat 10:88.

Yeatman JD, Dougherty RF, Myall NJ, Wandell BA, Feldman HM (2012) Tract profiles of white matter properties: automating fiber-tract quantification. PLoS One 7:e49790.

Yeterian EH, Pandya DN (1998) Corticostriatal connections of the superior temporal region in rhesus monkeys. J Comp Neurol 399:384-402.

Zatorre RJ, Belin P, Penhune VB (2002) Structure and function of auditory cortex: music and speech. Trends Cogn Sci 6:37-46.

Zatorre RJ, Fields RD, Johansen-Berg H (2012) Plasticity in gray and white: neuroimaging changes in brain structure during learning. Nat Neurosci 15:528-536. 